\title{
Évaluation de la dose patient en scanographie pédiatrique dans deux hôpitaux universitaires à Yaoundé Cameroun
}

\author{
P. ONGOLO-ZOGO ${ }^{1 *}$, C. MPEKE MOKUBANGELE ${ }^{2}$, B. MOIFO ${ }^{3}$, \\ J. GONSU FOTSIN ${ }^{3}$
}

(Manuscrit reçu le $1^{\text {er }}$ février 2012, accepté le 9 mars 2012)

RÉSUMÉ Les pratiques de radioprotection des patients sont peu documentées en Afrique subsaharienne en raison de l'absence ou de la mise en cuvre approximative des lois et règlements qui y sont relatifs. Ce travail investigue la justification et l'optimisation en scanographie pédiatrique dans deux hôpitaux universitaires de Yaoundé au Cameroun. L'enquête rétrospective portait sur 171 examens chez des enfants de 0 à 15 ans pendant un trimestre. Les données collectées à l'aide d'une fiche adaptée du modèle de l'Institut de radioprotection et de sureté nucléaire d'évaluation dosimétrique en scanographie ont été confrontées aux référentiels de la Société française de radiologie et de la Société francophone d'imagerie pédiatrique et prénatale. La conformité de chaque examen a été analysée et les valeurs d'index de dose scanographique et de produit dose longueur (PDL) ont été comparées aux niveaux de référence diagnostiques (NRD) des référentiels ci-dessus. Si tous les examens étaient justifiés, les protocoles, non affichés, étaient variables selon les équipes et les services. La tension était conforme dans $87,7 \%$ des cas et $15,2 \%$ des PDL étaient supérieurs aux NRD pour les scanners thoraciques et abdominaux en mode spiralé et cérébraux en mode séquentiel. Un affichage des protocoles optimisés est nécessaire pour améliorer les pratiques dans ces services avec une rotation importante de techniciens et résidents de radiologie.

ABSTRACT Evaluating pediatric patient dose during computed tomography in two University Teaching Hospitals in Yaoundé - Cameroun.

The practices related to patient radiation protection are poorly documented in subSaharan Africa because of the lack and/or weak enforcement of the related legislative and regulatory frameworks. This study investigates the justification and optimization of pediatric CT scans in two university teaching hospitals in Yaoundé in Cameroon. 171 CT scans in pediatric patients below 15 years of age performed during one trimester were surveyed. The data collection form was adapted from a dosimetric assessment sheet from the French Institut de radioprotection et de sureté nucléaire. The guidelines were those of the Société française de radiologie and the Société francophone d'imagerie pédiatrique et prénatale. Each CT scan was assessed and the CT dose index and dose length product were compared with the Diagnostic Reference Levels (DRLs) of the above-mentioned guidelines. While all the CT exams were justified, protocols were not readily available and varied amongst teams and

\footnotetext{
Département d’Imagerie Médicale et Radiothérapie, Faculté de Médecine et des Sciences Biomédicales, Université de Yaoundé 1, Yaoundé, Cameroun.

Service de Radiologie et Imagerie Médicale, Hôpital Central de Yaoundé, Yaoundé, Cameroun.

Service de Radiologie et Imagerie Médicale - Hôpital Gynéco-Obstétrique et Pédiatrique de Yaoundé, Yaoundé, Cameroun.
} 


\begin{abstract}
units. The tension conformed in $87.7 \%$ of cases and $15.2 \%$ of DLPs were higher than the DRL for thoracic and abdominal CT scans performed under helical mode and brain CT scans performed sequentially. Readily available optimal CT protocols for children are urgent to improve upon the pediatric CT practices in these two units with a high turn-over rate of technologists and radiology residents.
\end{abstract}

Keywords: pediatric imaging, patient radiation protection / computed tomography (CT) / practice assessment / Cameroon

\title{
1. Introduction
}

L'exploration scanographique représente désormais une part prépondérante des expositions diagnostiques aux rayons $\mathrm{X}$ et plus du tiers des expositions médicales (Brenner et Hall, 2007). Le prix à payer pour cette évolution technologique et l'amélioration subséquente de l'information diagnostique est l'augmentation des doses délivrées aux patients, notamment les enfants. En effet, la plus longue espérance de vie de ces derniers et leur plus grande susceptibilité accroissent le risque de survenue des effets délétères des radiations ionisantes quand l'exposition survient au cours de la première décade de la vie (IAEA, 2011 ; Valentin, 2007 ; Geleijns, 2010). Fréquemment, dans les services de radiologie générale les paramètres techniques optimisés pour les adultes sont appliqués aux enfants alors que, à paramètres équivalents, la dose délivrée à un enfant est 2,5 fois supérieure à celle délivrée à l'adulte (Paterson et al., 2001).

Les normes et standards internationaux (IAEA, 2011; Valentin, 2007; Geleijns, 2010) et les cadres règlementaires européen (Conseil de l'union européenne, 1997) et français (Mabille et al., 2005 ; SFIPP, 2006 ; Brisse et Aubert, 2009; SFR, 2009; IRSN, 2010) exigent que les équipements, les accessoires et les procédures soient adaptés à la pratique de l'imagerie pédiatrique : tout examen doit être justifié par son apport diagnostique en relation avec l'irradiation, sa réalisation doit être optimale c'est-à-dire conforme au principe ALARA, As Low As Reasonably Achievable, et les doses délivrées doivent être régulièrement évaluées pour confrontation aux niveaux de référence diagnostiques, ceux-ci ne doivent pas être dépassés sans justification. Les NRD sont des indicateurs de la qualité des pratiques permettant à chacun de situer sa pratique par rapport à l'ensemble de la profession et de corriger d'éventuels écarts. Les grandeurs utiles pour l'évaluation de la dose délivrée sont l'index de dose scanographique dans le volume, IDSV (ou CTDI ${ }_{\mathrm{vol}}$ ) et le Produit dose longueur (Boyer et al., 2002; Cordoliani, 2002; Cordoliani \& Boyer, 2004). Tout scanographe devrait fournir une estimation moyenne de l'IDSV en fonction des paramètres de chaque acquisition et le PDL par acquisition; le PDL cumulé est obligatoirement indiqué dans le compte rendu (Brisse et al., 2007). Le cadre référentiel de la radioprotection des patients en scanographie est extensivement 
décrit. En France, l'Institut de radioprotection et de sûreté nucléaire, IRSN, et la Société francophone d'imagerie pédiatrique et prénatale, SFIPP, ont publié des NRD pédiatriques (SFIPP, 2006; Brisse et Aubert, 2009). Les guides des indications et des procédures des examens d'imagerie répondent également à l'exigence de justification et d'optimisation avec en particulier chez les enfants l'obligation de substituer le scanner par l'échographie ou l'IRM chaque fois que possible (SFIPP, 2006; SFR, 2009). Différentes options sont offertes pour la conduite optimale d'un scanner justifié en pratique pédiatrique, notamment l'opportunité d'une acquisition multiphasique, la limitation du volume exploré, l'adaptation des paramètres selon l'âge, l'organe exploré et le type et la taille des lésions recherchées; l'utilisation de la tension et de la charge les plus basses compatibles avec une bonne qualité de l'image : kilovoltage entre 80 et $120 \mathrm{kV}$, réduction de la charge en agissant sur l'intensité et/ou le temps de rotation et/ou le pitch (Hollingswoth et al., 2003 ; Singh et al., 2009 ; Strauss et al., 2010).

À l'opposé, dans les pays d'Afrique subsaharienne, les cadres législatifs et règlementaires sont soit inexistants soit mis en œuvre de manière approximative et les pratiques de radioprotection des patients sont peu documentées alors que le parc scanographique s'accroît de manière constante depuis une décennie (Muhogora et al., 2008, 2010 ; Ongolo-Zogo et al., 2011). Dans le cadre d'un projet d'amélioration des pratiques de radioprotection, ce travail avait pour objectif d'évaluer, en termes dosimétriques, la pratique scanographique pédiatrique à Yaoundé au Cameroun.

\section{Population et méthodes}

Cette enquête d'évaluation des pratiques professionnelles s'est déroulée dans les services de radiologie et d'imagerie médicale de l'Hôpital central, HCY, et de l'Hôpital gynéco-obstétrique et pédiatrique de Yaoundé, HGOPY, équipés d'un scanographe de marque Siemens Somatom Duo double barrettes installé en juillet 2007 et d'un scanographe de marque Neusoft mono barrette installé en février 2007. Les deux scanographes sont dotés de protocoles pour enfants et offrent la possibilité de modifier le pitch, la tension et la charge. Ces deux services d'imagerie générale assurent les deux tiers de l'activité d'imagerie pédiatrique dans la ville de Yaoundé peuplée d'environ 1,5 million d'habitants. Ils sont centres de formation de médecins radiologistes et de techniciens d'imagerie médicale.

La population d'étude était constituée de tous les enfants âgés de 0 à 15 ans examinés au scanner entre décembre 2010 et février 2011. La fiche de recueil de données, inspirée d'un modèle IRSN pour l'évaluation dosimétrique en scanographie, comportait les rubriques suivantes : hôpital, type de scanographe, identification du patient, examen pratiqué, indications et résultats obtenus, 
paramètres d'examen-type et nombre d'acquisition, kilovoltage en $\mathrm{kV}$, charge en $\mathrm{mAs}$, épaisseur en millimètres, nombre de coupes, pitch et doses délivrées, IDS, IDSV et PDL (voir annexe).

Le recensement des examens pédiatriques dans les registres d'activité a permis la collecte des données d'identification avant celle des paramètres techniques à partir de la mémoire centrale du scanographe ou des cédéroms d'archivage. Les comptes rendus d'examens et certains bulletins de demande d'examen conservés ont fourni les détails relatifs aux indications et aux résultats des examens. Les fiches ont été vérifiées par un second enquêteur. Par ailleurs, la disponibilité en salle des protocoles ainsi que la liste des techniciens et médecins intervenant au scanner ont été vérifiées.

Les fréquences des variables qualitatives ont été calculées; les variables quantitatives ont fait l'objet d'un calcul de moyennes, d'écarts-types et de médianes. Les valeurs totales de PDL ont été calculées pour chaque individu. Chaque examen a été analysé pour la conformité de l'indication et des paramètres techniques aux référentiels SFR et SFIPP. La comparaison des valeurs moyennes de PDL par type d'examen et par service de cette population avec les NRD de la SFIPP a été effectuée au moyen du test $t$ de Student.

Cette étude a été autorisée par les autorités hospitalières et le comité national d'éthique.

\section{Résultats}

\subsection{Patients}

Le tableau I décrit les caractéristiques des 171 enfants $(88 \mathrm{M} / 83 \mathrm{~F})$ inclus. 33 $(19,3 \%)$ étaient âgés de 0 à 1 an, $49(28,7 \%)$ de 1 à 5 ans et $89(52 \%)$ de 5 à 15 ans. Les scanners pédiatriques représentaient 171/1220 scanners (14\%) effectués au cours de cette période : 7,3 \% à HCY et 20,7 \% à HGOPY.

\subsection{Justification}

Le tableau II illustre les motifs cliniques qui étaient tous justifiés dans ce contexte en raison de l'inaccessibilité physique et financière d'une IRM. En théorie, 90 examens $(52,6 \%)$ pouvaient être substitués par une IRM. Le tableau III indique les conclusions des scanners dont $31,6 \%$ étaient normaux et $67,2 \%$ pathologiques. 
TABLEAU I

Répartition des examens en fonction du sexe et de la tranche d'âge. Distribution of exams in relation to sex and age interval.

\begin{tabular}{cccccccccccc}
\hline & & \multicolumn{2}{c}{ Crâne } & \multicolumn{2}{c}{ Thorax } & \multicolumn{2}{c}{ Abdomen } & \multicolumn{2}{c}{ Pelvis } & \multicolumn{2}{c}{ Rachis } \\
& & $\mathbf{n}$ & $\%$ & $\mathbf{n}$ & $\%$ & $\mathbf{n}$ & $\%$ & $\mathbf{n}$ & $\%$ & $\mathbf{n}$ & $\%$ \\
\hline Sexe & Masculin & 80 & 53,3 & 1 & 25 & 5 & 62,5 & 1 & 50 & 1 & 14,3 \\
& Féminin & 70 & 46,7 & 3 & 75 & 3 & 37,5 & 1 & 50 & 6 & 85,7 \\
Age (années) & {$[\mathbf{0 ; 1 ]}$} & 31 & 20,7 & 0 & 0 & 2 & 25,0 & 0 & 0 & 0 & 0 \\
& $\mathbf{1 1 ; 5}]$ & 42 & 28,0 & 2 & 50 & 4 & 50,0 & 1 & 50 & 0 & 0 \\
& $\mathbf{] 5 ; 1 5}]$ & 77 & 51,3 & 2 & 50 & 2 & 25,0 & 1 & 50 & 7 & 100 \\
& Total & $\mathbf{1 5 0}$ & $\mathbf{1 0 0}$ & $\mathbf{4}$ & $\mathbf{1 0 0}$ & $\mathbf{8}$ & $\mathbf{1 0 0}$ & $\mathbf{2}$ & $\mathbf{1 0 0}$ & $\mathbf{7}$ & $\mathbf{1 0 0}$ \\
\hline
\end{tabular}

TABLEAU II

Indications cliniques.

Clinical indications.

\begin{tabular}{lcc}
\hline Renseignements & $\mathbf{n}$ & $\%$ \\
\hline Traumatisme crânien & 39 & 22,8 \\
Comitialité & 27 & 15,8 \\
Malformations cérébrales & 22 & 12,8 \\
Tumeurs & 20 & 11,6 \\
Retard Psychomoteur & 20 & 11,6 \\
Céphalées & 19 & 11,1 \\
Hémiparésie, Hémiplégie & 14 & 8,3 \\
Trouble de l'équilibre & 4 & 2,4 \\
Rachialgie & 4 & 2,4 \\
Pneumopathie & 2 & 1,2 \\
Total & $\mathbf{1 7 1}$ & $\mathbf{1 0 0 , 0}$ \\
\hline
\end{tabular}

TABLEAU III

Conclusions des scanners.

CT scans conclusions.

\begin{tabular}{lcc}
\hline Résultats & $\mathbf{n}$ & \% \\
\hline Diverses anomalies encéphaliques & 76 & 43,9 \\
Lésions intra crâniennes traumatiques & 24 & 14,3 \\
Pathologies abdomino-pelviennes & 8 & 4,8 \\
Pathologies rachidiennes & 4 & 2,4 \\
Pneumopathies & 3 & 1,8 \\
Normaux & $\mathbf{5 4}$ & $\mathbf{3 1 , 6}$ \\
Données manquantes & 2 & 1,2 \\
Total & $\mathbf{1 7 1}$ & $\mathbf{1 0 0 , 0}$ \\
\hline
\end{tabular}


TABLEAU IV

Paramètres techniques utilisés pour les deux scanners pour différents examens. Technical parameters applied to both CT scanners for different exams.

\begin{tabular}{|c|c|c|c|c|c|}
\hline Scanner Siemens Somatom Duo (HCY) & Crâne & Abdomen & Thorax & \multicolumn{2}{|c|}{ Rachis } \\
\hline Haute tension $(\mathrm{kV})$ & $110-130$ & 110 & 110 & \multicolumn{2}{|c|}{110} \\
\hline Charge par coupe (mAs) & $162-260$ & $26-38$ & $25-38$ & \multicolumn{2}{|c|}{80} \\
\hline Épaisseur de coupe (mm) & $4-8$ & $2,5-6$ & 2,5 & \multicolumn{2}{|c|}{2,5} \\
\hline Pitch & NA & 2 & 2 & \multicolumn{2}{|c|}{1} \\
\hline Temps de rotation (s) & 1,5 & 0,8 & 0,8 & \multicolumn{2}{|c|}{1} \\
\hline Type d'acquisition & $\mathrm{S}$ & $\mathrm{H}$ & $\mathrm{H}$ & \multicolumn{2}{|c|}{$\mathrm{H}$} \\
\hline Scanner Neusoft (HGOPY) & Crâne & Abdomen & Thorax & Rachis & Pelvis \\
\hline Haute tension $(\mathbf{k V})$ & 120 & 120 & 120 & 120 & 120 \\
\hline Charge par coupe (mAs) & $150-160$ & $150-160$ & 155 & $150-155$ & 150 \\
\hline Épaisseur de coupe (mm) & 5 & $5-10$ & $1-5$ & 3 & 5 \\
\hline Pitch & 1 & 1 & 1 & 1 & 1 \\
\hline Temps de rotation (s) & 1 & 1 & 1 & 1 & 1 \\
\hline Type d'acquisition & $\mathrm{H}$ & $\mathrm{H}$ & $\mathrm{H}$ & $\mathrm{H}$ & $\mathrm{H}$ \\
\hline
\end{tabular}

$\mathrm{kV}$ : Kilovolt ; mAs : milliampère seconde ; S : séquentiel ; H : hélicoïdal ; HCY : Hôpital Central ; HGOPY : Hôpital Gynéco-Obstétrique et Pédiatrique ; NA : non applicable

\subsection{Paramètres d'examen}

Le tableau IV décrit les paramètres techniques utilisés lors des examens. 78,9\% des acquisitions étaient en mode hélicoïdal contre $21,1 \%$ en mode séquentiel. 127 examens ont été réalisés à $120 \mathrm{kV}, 30$ à $110 \mathrm{kV}$ et 14 à $130 \mathrm{kV}$. Le temps d'acquisition et de rotation du tube était de $1 \mathrm{~s}$ pour 129 examens $(75,4 \%), 1,5 \mathrm{~s}$ pour 36 examens $(21,1 \%)$ et 0,8 s pour 6 examens $(3,5 \%)$. La charge variait entre 25 et $260 \mathrm{mAs}$ avec une moyenne de $163 \pm 40$ et une médiane de 155 .

\subsection{Conformité aux référentiels SFR et SFIPP}

Le tableau IV indique les paramètres techniques selon les services. La tension était conforme pour $87,7 \%$ des examens et la charge était conforme pour $91,2 \%$ des examens. 84,8\% des PDL étaient inférieurs ou égaux aux NRD de la SFIPP. Le dépassement des NRD dans $12 \%$ des scanners cérébraux était en relation avec une tension inappropriée $(9,3 \%)$ ou une charge élevée $(10 \%)$. Le survoltage expliquait le dépassement observé dans $25 \%$ d'examens thoraciques et $62,5 \%$ d'examens abdominaux. De manière spécifique, les examens cérébraux à l'HCY étaient réalisés à $130 \mathrm{kV}$, à $240 \mathrm{mAs}$ en acquisition séquentielle avec temps de rotation de $1,5 \mathrm{~s}$. Le dépassement de NRD pour les examens du thorax et abdomen à l'HGOPY était associé à une haute tension supérieure à $110 \mathrm{kV}$ et une charge supérieure à $150 \mathrm{mAs}$ pour les deux tranches d'âge les plus jeunes. Le tableau $\mathrm{V}$ indique la haute tension, la charge ainsi que les IDSV et PDL observées et la comparaison avec les NRD de la SFIPP. 
ÉVALUATION DE LA DOSE PATIENT EN SCANOGRAPHIE PÉDIATRIQUE

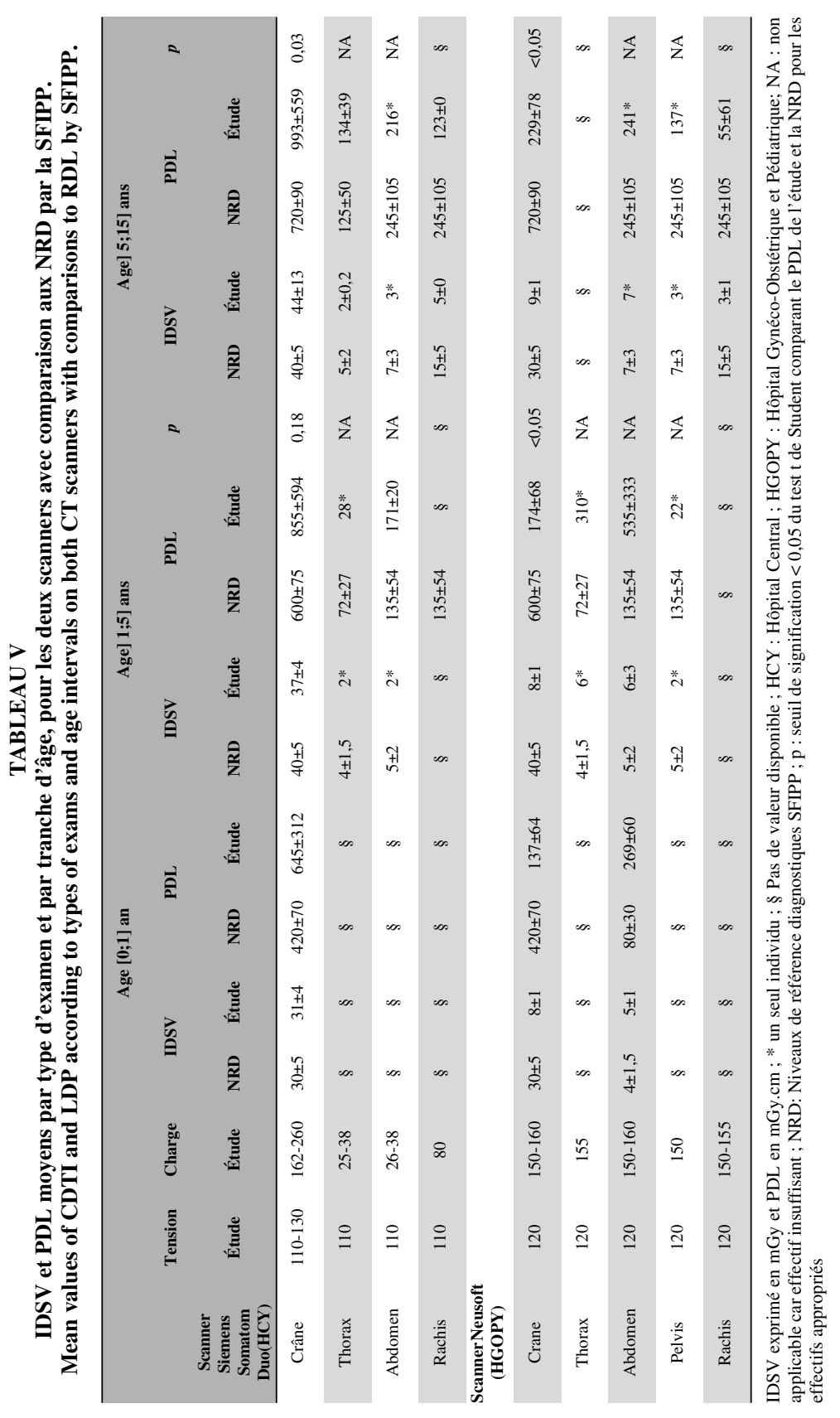

RADIOPROTECTION - VOL. 47 - N 4 (2012) 


\section{Discussion}

Ce travail fournit un instantané des pratiques de radioprotection des patients dans les deux centres les plus actifs en termes de scanographie pédiatrique au Cameroun: ces pratiques ne sont pas optimales. L'absence d'affichage de protocoles optimisés dans ces services avec une grande rotation de techniciens et de résidents de radiologie explique en partie les écarts observés.

Malgré l'existence d'une loi encadrant la radioprotection et d'une Agence nationale de radioprotection (Ongolo-Zogo et al., 2011), l'absence de cadre règlementaire de la protection des patients justifie le recours aux référentiels SFR et SFIPP. Si les efforts de l'AIEA portent des fruits avec la création d'une agence de radioprotection, la rareté des ressources contraint à la mise en œuvre approximative des conventions internationales qui s'imposent en l'absence de cadre national (Muhogora et al., 2008, 2010).

La proportion des examens pédiatriques dans ces centres non équipés d'IRM s'inscrit dans la dynamique croissante de la scanographie pédiatrique passée de $4 \%$ en 1998 à $11 \%$ en 2004 (Brenner et Hall, 2007). Trois scanners cérébraux sur quatre étaient substituables par une IRM en dehors des urgences traumatiques crânio-cérébrales (SFIPP, 2006). Si les scanners cérébraux étaient justifiés par l'inaccessibilité économique et géographique à l'IRM, les NRD étaient dépassés en raison du mode séquentiel sans adaptation de la charge notamment la nonréduction du temps de rotation ou du courant. Le faible niveau des connaissances en radioprotection explique également la proportion non négligeable d'examens en marge des recommandations alors que les scanographes offraient l'option d'ajuster les paramètres de tension et de charge et des protocoles «enfants » (Ongolo-Zogo et al., 2011). La réduction de la tension en dessous de $120 \mathrm{kV}$ pour le crâne et de la charge entre 125 et 150 mAs est démontrée efficace pour réduire de l'ordre de $40 \%$ la dose délivrée sans altérer la qualité diagnostique des images (Hollingswoth et al., 2003; Singh et al., 2009; Strauss et al., 2010). La vulnérabilité particulière du thorax en raison des nombreux organes sensibles tels la moelle osseuse, les poumons, la thyroïde et les seins exige une adaptation des paramètres selon le poids (Hollingswoth et al., 2003). Comme observé ailleurs, la diversité des protocoles techniques fait varier la dose délivrée d'un facteur 1 à 4 y compris avec les mêmes scanographes (Hollingswoth et al., 2003). Les charges appliquées dans ce groupe étaient largement au-dessus des 50 à $80 \mathrm{mAS}$ testés comme n'altérant pas la qualité diagnostique de l'image pour les explorations abdominales et thoraciques (Singh et al., 2009).

Si l'affichage de l'IDSV et du PDL aide à contrôler l'optimisation des protocoles et à comparer les pratiques, il nous faut admettre que ces valeurs sousestiment la dose délivrée aux enfants malgré l'existence de protocoles pédiatriques adaptés selon les tranches d'âge ou de poids (Paterson et al., 2001 ; Singh et al., 
2009). Les systèmes de modulation automatique de la dose, AEC, Automatic Exposure Control, proposés par certains constructeurs permettent de réduire davantage les doses délivrées (Strauss et al., 2010).

Au moment où le parc scanographique au Cameroun s'étend vers les hôpitaux régionaux avec une vocation d'imagerie générale, il est urgent que les centres universitaires travaillent à l'optimisation des protocoles pédiatriques. En affichant et en appliquant ces derniers, il est possible d'éviter une irradiation indue des jeunes enfants et l'évaluation périodique des doses délivrées devraient devenir une activité routinière.

Ce travail initial a permis la conception d'une intervention comportant la sensibilisation de l'ensemble des personnels des hôpitaux universitaires de Yaoundé, notamment les résidents, l'affichage des protocoles modifiés pour les enfants - réalisation hélicoïdale préférentielle, réduction de la tension à $110 \mathrm{kV}$, du temps de rotation à $0,8 \mathrm{~s}$ dont les effets seront évalués à 6,12 et 18 mois en se focalisant sur les scanners crânio-cérébraux et thoraciques.

ANNEXE : FICHE DE COLLECTE DES DONNEES

\begin{tabular}{|l|l|}
\hline LIEU/ ETABLISSEMENT & MARQUE ET MODELE DU SCANNER \\
\hline HCY & \\
\hline HGOPY & \\
\hline
\end{tabular}

IDENTIFICATION DU PATIENT

\begin{tabular}{|l|l|l|l|}
\hline Code & No de l'examen & Age & Sexe \\
\hline & & & \\
\hline
\end{tabular}

TYPE D'EXAMEN SCANOGRAPHIQUE

\begin{tabular}{|l|l|l|l|l|l|}
\hline Date & Crane & Thorax & Abdomen & Pelvis & Rachis \\
\hline & & & & & \\
\hline
\end{tabular}

INDICATIONS ET RESULTATS

\begin{tabular}{|l|l|}
\hline RENSEIGNEMENTS CLINIQUES & RESULTATS \\
\hline & \\
\hline
\end{tabular}

\section{PARAMETRES D'EXAMEN ET DOSES DELIVREES}

\begin{tabular}{|c|c|c|c|c|c|c|c|c|}
\hline $\begin{array}{c}\text { Acquisition } \\
\text { Type : S ou H Nombre : }\end{array}$ & kV & mA & s & $\begin{array}{c}\text { Epaisseur } \\
\text { coupe }\end{array}$ & $\begin{array}{c}\text { Nombre } \\
\text { Coupe }\end{array}$ & Pitch & $\begin{array}{c}\text { IDSV } \\
(\mathbf{m G y})\end{array}$ & $\begin{array}{c}\text { PDL } \\
(\mathbf{m G y} . c m)\end{array}$ \\
\hline & & & & & & & & \\
\hline & & & & & & & & \\
\hline
\end{tabular}




\section{RÉFÉRENCES}

Boyer B., Le Marec E., Hauret L., Jouan E., Cordoliani Y.S. (2002) Principes du scanner hélicoïdal : irradiation, Feuillets Radiologie 42, 239-247.

Brenner D.J., Hall E.J. (2007) Computed tomography: an increasing source of radiation exposure, $N$. Engl. J. Med. 357, 2277-84.

Brisse H., Sirinelli D., Chateil J.-F. et al. (2007) Inscrire la dose d'exposition dans les comptesrendus radiologiques : pourquoi ? comment ? J. Radiol. 88, 411-414.

Brisse H., Aubert B. (2009) Niveaux d'exposition en tomodensitométrie multicoupes pédiatrique : résultats de l'enquête dosimétrique SFIPP/IRSN 2007-2008, J. Radiol. 90, 207-215.

Conseil de l'union européenne (1997) Directive 97/43 EURATOM du Conseil du 30 juin 1997 relative à la protection sanitaire des personnes contre les dangers des rayonnements ionisants lors d'exposition à des fins médicales, Bruxelles, Belgique.

Cordoliani Y.S. (2002) Grandeurs et unités en radioprotection, Feuillets Radiologie 42, 248-252.

Cordoliani Y.S., Boyer B. (2004) Radioprotection: doses délivrées au patient en scanographie, Feuillets Radiologie 44, 139-145.

Geleijns J. (2010) Optimisation of radiation protection for pediatric and adult patients in radiography and computed tomography, Proceedings Third European IRPA Congress 2010, Helsinki, Finland. pp. 2972-80.

Hollingsworth C, Frush DP, Cross M, Lucaya J. (2003) Helical CT of the body: a survey of techniques used for pediatric patients, Am. J. Roentgenol. 180, 401-406.

IAEA (2011) Radiation protection of patients, CT optimization. Accès le 23 janvier 2012 sur : https://rpop.iaea.org/RPOP/RPoP/Content/InformationFor/HealthProfessionals/1_Radiology/ ComputedTomography/CTOptimization.htm.

IRSN (2010) Rapport DRPH n²010-15. Analyse des données relatives à la mise à jour des niveaux de référence diagnostiques en radiologie et en médecine nucléaire. Bilan 2007-2008. Paris, France.

Mabille M., Beauvais-March H., Rehel J.-L., Kalifa G. (2005) Évaluation des doses d'irradiation aux organes en scanographie pédiatrique, J. Radiol. 86, 487-491

Muhogora W.E., Ahmed N.A., Almosabihi A., Alsuwaidi J.S., Beganovic A., Ciraj-Bjelac O. et al. (2008) Patient doses in radiographic examinations in 12 countries in Asia, Africa, and Eastern Europe: initial results from IAEA projects, Am. J. Roentgenol. 190, 1453-1461.

Muhogora W.E., Ahmed N.A., Almosabihi A., Alsuwaidi J.S., Beganovic A., Ciraj-Bjelac O. et al. (2010) Paediatric ct examinations in 19 developing countries: frequency and radiation dose, Radiat. Prot. Dosim. 140, 49-58.

Ongolo-Zogo P., Nguehouo B.S., Yomi J., Nko'o Amvene S. (2011) Connaissances en matière de radioprotection : enquête auprès des personnels des services hospitaliers de radiodiagnostic, radiothérapie et médecine nucléaire à Yaoundé. Communication orale Journées Françaises de Radiologie 21-25 Octobre 2011, Paris, France.

Paterson A., Frush D.P., Donnelly L.F. (2001) Helical CT of the body: are settings adjusted for pediatric patients? Am. J. Roentgenol. 176, 297-301.

SFIPP (2006) Critères de qualité et optimisation des doses en scanographie chez l'enfant. Accès le 23 janvier 2012 sur http://www.sfipradiopediatrie.org/images/stories/docs_telechargement/guideprocedurestdmpe di06.pdf.

SFR (2009) Guide pratique à l'usage des médecins radiologues. Accès le 23 janvier 2012 sur http://www.sfrnet.org/sfr/societe/2-publications/publications-sfr/01-Guides2009/index.phtml

Singh S., Kalra M.K., Moore M.A., et al. (2009) Dose reduction and compliance with pediatric CT protocols adapted to patient size, clinical indication, and number of prior studies, Radiology 252, 200-208.

Strauss K.J., Goske M.J., Kaste S.C., et al. (2010) Image gently: ten steps you can take to optimize image quality and lower CT dose for pediatric patients, Am. J. Roentgenol. 194, 868-73.

Valentin J. (2007) Managing patient dose in multi-detector computed tomography, Ann. ICRP 37, 1-79. 